\title{
DRIVER NAVIGATION AND SAFETY ERRORS IN ALZHEIMER'S DISEASE AND STROKE
}

\author{
Ergun Y. Uc, Jennifer L. Smothers, Qian Shi, Matthew Rizzo \\ College of Medicine, Engineering, and Public Health \\ Public Policy Center \\ University of Iowa \\ Iowa City, IA, USA \\ E-mail: ergun-uc@uiowa.edu
}

\begin{abstract}
\section{Objective}

Assess navigation and safety errors during a route-following task in drivers with Alzheimer's disease (AD) and stroke.
\end{abstract}

\section{Background}

Safe automobile driving requires drivers to perform multiple competing tasks while remembering and implementing correct road rules and routes. Neurologic disorders such as AD and stroke can impair these abilities, thereby increasing the risk of navigation errors and safety errors that may lead to collisions.

\section{Design/Methods}

One hundred and one legally licensed active drivers participated: 7 (mean age $\pm \mathrm{SD}=75.3 \pm 5.0$ ) had probable AD (NINCDS criteria) of mild severity, $19(61.8 \pm 10.5)$ had cerebral lesions mostly due to stroke, and $75(69.7 \pm 5.2)$ were neurologically normal. All drivers participated on a battery of visual and cognitive tests. None had active, confounding medical or psychiatric conditions. Median visual acuity was 20/20, and no driver had acuity below 20/32-2. Each driver was required to learn and recite correctly a brief set of verbal directions on a navigation task administered as part of an hour-long drive conducted in an experimental vehicle named ARGOS. Instrumentation and sensors aboard ARGOS recorded driver speed, steering, braking and acceleration at $10 \mathrm{~Hz}$, in synch with 4 video views of the driver and the roadway. Dependent measures were number of: 1) trials to correctly memorize the route prior to the drive, 2) correct turns, 3) times lost (i.e., unable to resume the correct route without prompting from the examiner), and 4) at-fault safety errors. Safety errors were further classified into categories of perception/attention, memory, and other, based on video analysis, driver self-report, and questionnaire data.

\section{Results}

The drivers with $\mathrm{AD}$ and stroke required more trials to learn the route correctly $(\mathrm{P}<0.01$ and $\mathrm{P}=0.04$, respectively, Wilcoxon Rank Sum) and made fewer correct turns than the neurologically normal controls $(\mathrm{P}=0.02$ and $\mathrm{P}<0.001$, respectively). Drivers with stroke got lost more often $(\mathrm{P}=0.006)$ than controls. Turn errors in patients with $\mathrm{AD}$ were associated with perception/ 
attention problems ( $\mathrm{P}=0.011$, Fisher's exact test). Further, the drivers with $\mathrm{AD}$ and stroke made more at-fault safety errors than normal controls during the route following task $(\mathrm{P}=0.011$ and $\mathrm{P}=0.014$, respectively). These errors mainly comprised illegal lane boundary crossings of varying severity. Number of safety errors and number of correct turns did not correlate with the ability to learn the route.

\section{Conclusions}

Drivers with AD and stroke made more errors than neurologically normal drivers on a routefollowing task that placed demands on driver memory, attention, and perception. These demands probably increased the cognitive load during driving, which may explain the higher number of safety errors we observed in these drivers. Getting lost can conceivably increase driver anxiety due to uncertainty of location, extended searching for landmarks, or attempts to read maps or signs, and further divert cognitive resources from key driving tasks in drivers whose baseline cognitive resources are limited. The current results indicate that real-world tests of driver fitness can benefit from adding controlled challenges of memory, perception, and attention during driving, especially in cognitively impaired populations such as patients with AD, Parkinson's disease, and stroke.

\section{Acknowledgments}

This research was supported by NIA AG 17717 and NIA AG 15071. 